\title{
Magnaporthe puts a ring on it
}

septins are

important for

positioning

the proteins

responsible for

generating the

penetration

peg ... to the

centre of the

appressorium

pore
The fungus Magnaporthe oryzae, the causative agent of rice blast disease, infects leaves using a specialized dome-shaped cell known as an appressorium. The melanin-rich cell wall of the appressorium enables it to generate turgor pressure of $8.0 \mathrm{MPa}$, sufficient to drive a narrow extension known as a penetration peg from the base of the cell through the tough cuticle of the plant leaf. Writing in Science, Dagdas et al. now describe a ring-shaped structure at the base of the appressorium that is formed by septins, F-actin and other cytoskeletal components and provides the rigidity and negative membrane curvature needed for protrusion of the penetration peg into the rice leaf.

To investigate what happens to the fungal cytoskeleton in the appressorium during plant cell invasion, Dagdas et al. used an actin-binding protein fused to RFP (LifeAct-RFP) to visualize the actin cytoskeleton as the appressorium matured. They observed the formation of an extensive toroidal network of F-actin at the base of the cell surrounding the appressorium pore, an area of the cell surface that lacks cell wall and from which the penetration peg emerges. Complex networks of F-actin in other cytoskeletal systems are organized by septins, a family of small morphogenetic GTPases. Accordingly, the authors identified five genes in the $M$. oryzae genome that encode putative septins, four of which exhibited sequence similarity to the core septins found in Saccharomyces cerevisiae and complemented temperature-sensitive yeast mutants of their respective homologues. The authors generated GFP-tagged versions of the four septins and observed that each formed part of a $5.9 \mu \mathrm{m}$ ring that colocalized with the F-actin network. In isogenic mutants lacking any one of the four septins, the other septins and F-actin mislocalized and failed to form the ring structure, suggesting that the four septins act cooperatively to organize the F-actin network in the appressorium. Importantly, loss of the ring structure in these mutants correlated with a loss of pathogenicity, and inoculation of a susceptible rice plant with each septin-null mutant caused either no symptoms or just small necrotic flecks indicative of abortive infection.

As well as acting as a scaffold for F-actin, septins have been shown to act as a barrier to lateral diffusion of membrane-associated proteins, for instance at the site of daughter cell budding in S. cerevisiae. In agreement with septins having such a role at the base of the appressorium, the authors found that Rvs167 (an inverse BAR (I-BAR) protein) and Las17 (a WASP-WAVE complex component) localized to the centre of the appressorium pore in wild-type $M$. oryzae but not in a mutant lacking one of the four septins. Interestingly, I-BAR and WASP-WAVE complex proteins are involved in generating the negative membrane curvature that is required for cellular protrusions. This suggests that, as well as organizing the ring structure that stabilizes the appressorium pore, septins are important for positioning the proteins responsible for generating the penetration peg, partitioning these proteins to the centre of the appressorium pore.

Taken together, these findings provide important insights into the mechanisms underlying the infection of leaves by M. oryzae and may provide new potential targets for preventing the spread of rice blast disease, which affects up to $30 \%$ of the world's rice harvest each year.

Andrew Jermy

ORIGINAL RESEARCH PAPER Dagdas, Y. F. Septin-mediated plant cell invasion by the rice blast fungus Magnaporthe oryzae. Science 336, 1590-1595 (2012)

FURTHER READING Wilson, R. A. \& Talbot, N. J. Under pressure: investigating the biology of plant infections by Magnaporthe oryzae. Nature Rev. Microbiol. 7, 185-195 (2009)

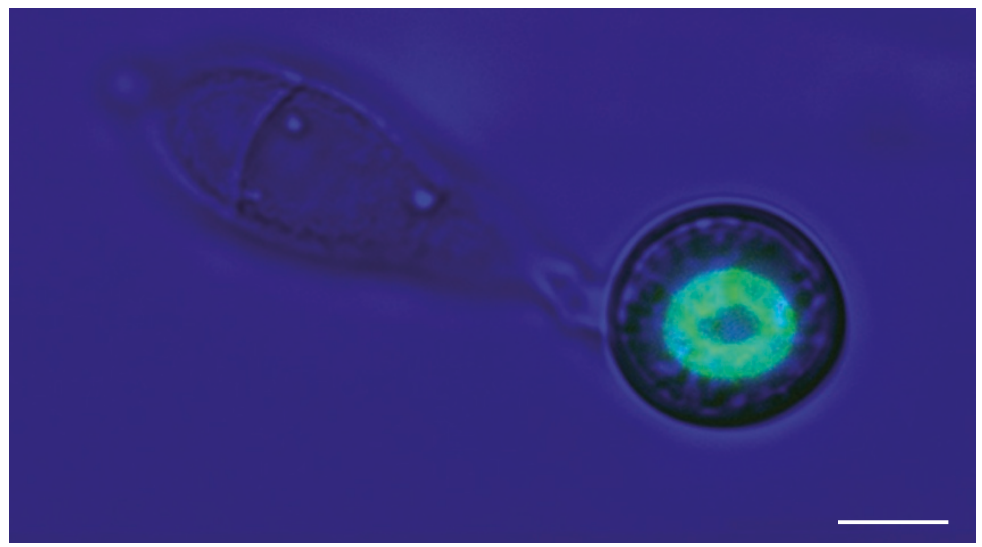

Micrograph showing the septin ring that forms at the base of the Magnaporthe oryzae appressorium; a Sep3-GFP fusion protein is visualized by laser excitation epifluorescence microscopy (scale bar represents $5 \mu \mathrm{m}$ ). The dome-shaped appressorium forms at the end of a short germ tube following germination of the three-celled, tear-drop-shaped spore. Image courtesy of N. J. Talbot, University of Exeter, UK. 